\title{
PLASTIC FLOW OF $\gamma$-TIAI-BASED POLYSYNTHETICALLY TWINNED CRYSTALS: MICROMECHANICAL MODELING AND EXPERIMENTAL VALIDATION
}

\author{
R. LEBENSOHN ${ }^{1}$, H. UHLENHUT ${ }^{2} \uparrow$, C. HARTIG ${ }^{2}$ and H. MECKING ${ }^{2}$ \\ ${ }^{1}$ IFIR (UNR-CONICET), 27 de Febrero 210 Bis, 2000 Rosario, Argentina and ${ }^{2}$ Technical University \\ Hamburg-Harburg, 21071 Hamburg, Germany
}

(Received 9 February 1998; accepted 28 March 1998)

\begin{abstract}
A micromechanical model for the calculation of the plastic behavior of a lamellar structure is presented. This model is based on a rate-sensitive approach to describe the plasticity at the single crystal (lamella) level and on the relaxed constraints theory to account for the influence of the lamellar morphology on the overall plastic response of the structure. The equations for the cases of 2- and $N$-lamellae structures undergoing states of applied stress or strain rate are presented. The model is applied to a lamellar matrix-twin pair which is a simplified representation of a $\gamma$-TiAl polysynthetically twinned (PST) crystal. For this case, a morphology-based classification of the critical stresses of the $\gamma$-TiAl deformation systems is also presented. This model for PST plasticity is successfully validated by comparison with available experimental data. (C) 1998 Acta Metallurgica Inc. Published by Elsevier Science Ltd. All rights reserved.
\end{abstract}

\section{INTRODUCTION}

Due to intensive research, TiAl-based alloys have gained a great potential as high temperature structural materials. On the basis of experimental efforts, a technology has been developed which allows the first applications at the industrial scale [1-3]. Nevertheless, the interpretation of the available experimental data as well as the optimization of the experimental procedures require a better understanding of the basic mechanisms that determine the material properties. This necessity has given rise to an increasing modeling activity in this field. Micromechanical models of crystal and polycrystal plasticity are good candidates for this task. Nevertheless, these models should be refined in order to account for the complex microstructures of the TiAl alloys.

The $\gamma$-TiAl-based alloys exhibit a variety of different microstructures [4]. TiAl sheets, for example, have a homogeneous, fine grained, equiaxed microstructure after rolling which allows superplastic forming (SPF) $[1,2]$. During rolling and subsequent SPF, a texture develops and this causes a strong plastic anisotropy of the sheet [5]. If a good creep resistance of these alloys is required, the formation of lamellar microstructures should be favored [6-8]. These kind of microstructures can be obtained by appropriate heat treatments or just after casting [4,9]. Especially the as-cast material [10] and also the directionally solidified

$\dagger$ To whom all correspondence should be addressed. material [11] are expected to be highly anisotropic due to preferred orientations of the lamellar colonies. A lot of experimental work has been devoted to understanding the basic mechanisms of plastic deformation of polysynthetically twinned (PST) crystals. A PST crystal consists of a single set of lamellae (i.e. a unique direction of the normal to the lamellar planes can be defined). The crystallography, morphology and strong plastic anisotropy of PST crystals have been described in great detail (for a thorough discussion, see Refs [12-14]). A PST crystal is formed essentially by $\gamma$-TiAl lamellae (tetragonal $\mathrm{L}_{0}$ structure) and also by a few percents of $\alpha_{2}-\mathrm{Ti}_{3} \mathrm{Al}$ lamellae (hexagonal $\mathrm{D} 0_{19}$ structure). The orientation relationship between $\gamma$ - and $\alpha_{2}$-phases is $\{111\}_{\gamma} / /(0001)_{\alpha_{2}}$ and $\langle 1 \overline{1} 0\rangle_{\gamma} / /\langle 11 \overline{2} 0\rangle_{\alpha_{2}}$ with the basal plane of the $\alpha_{2}$-phase being parallel to the habit plane of the lamellae. In the $\gamma$-phase, due to its tetragonality, there are six different crystallographic orientations which fulfill the above orientation relations. This results in the so-called "domain structure" of twin lamellae which is sketched in Fig. 1. Each matrix lamella consists of three types of domains $\left(\mathrm{I}^{\mathrm{M}}, \mathrm{II}^{\mathrm{M}}\right.$ and $\left.\mathrm{III}^{\mathrm{M}}\right)$ which can be distinguished by a $120^{\circ}$ rotation around the [111] direction and the corresponding twin lamella consists of domains having the corresponding twinned orientations $\left(\mathrm{I}^{\mathrm{T}}, \mathrm{II}^{\mathrm{T}}\right.$ and $\left.\mathrm{III}^{\mathrm{T}}\right)$. Therefore, there are six possible orientations of these domains. Concerning the stacking sequence, a $\gamma$-lamella can have a lamellar boundary in common with a $\alpha_{2}$ lamella or with another $\gamma$-lamella of a twinned 


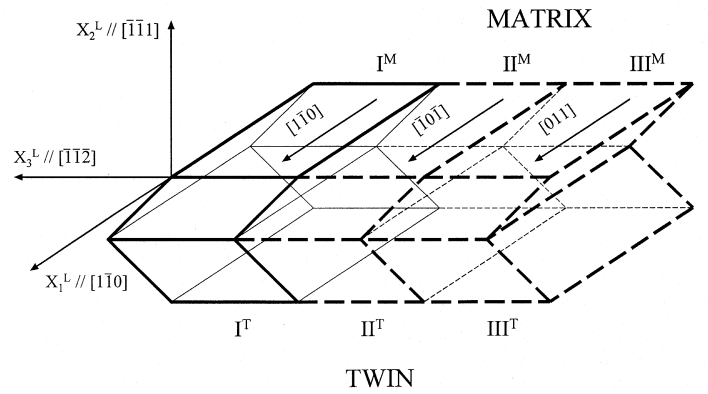

Fig. 1. Schematic matrix-twin pair of lamellae, representing the structure of a PST crystal. Each lamella contains three $\gamma$-domains (matrix: $\mathrm{I}^{\mathrm{M}}, \mathrm{II}^{\mathrm{M}}$ and $\mathrm{III}^{\mathrm{M}}$, twin: $\mathrm{I}^{\mathrm{T}}, \mathrm{II}^{\mathrm{T}}$ and III $^{\mathrm{T}}$ ) which share the $[\overline{11} 1]$-axis but can be distinguished by the orientation of the $\langle 110\rangle$ directions as indicated with arrows for the matrix lamellae. The simplified PST model consists of the domains $\mathrm{I}^{\mathrm{M}}$ and $\mathrm{I}^{\mathrm{T}}$ only, which are sketched with solid lines. The coordinate system indicates the definition of the "lamellar axes" reference frame for the matrix-twin pair which has been chosen to formulate the present model.

orientation. For typical $\gamma$-TiAl-based alloys, the latter is more frequently found.

Classical crystal and polycrystal plasticity models assume that at the single crystal level dislocation glide is the only available mechanism of plastic deformation. At the polycrystal level, both the simple approaches like the Taylor model [15] — which assumes homogeneous deformation in the whole polycrystal and disregards grain morphology-or the more sophisticated formulations like the relaxed constraints (RC) model [16] or the one-site viscoplastic self-consistent (VPSC) model [17, 18]—which allow differences in deformation from grain to grain and account for grain morphology effects - are based on the assumptions of homogeneous deformation inside the grains and of a perfectly disordered polycrystal (i.e. no crystallographic or morphologic correlations between neighboring grains).

In recent years, crystal and polycrystal plasticity models were improved in order to consider more complex types of intracrystalline deformation. Examples of this are the different efforts to model the role of deformation twinning $[19,20]$ and the effect of phase morphology and orientation relationship between phases in lamellar two-phase polycrystals [21]. Following similar lines, the aim of this paper is to describe the plastic behavior of a PST crystal taking explicitly into account the role of crystallography and morphology of the twin lamellae. In doing this, use is made of a rate-sensitive approach at the single crystal (lamella) level and of the RC theory at the PST level, respectively. It will be also shown that this model can be easily adapted to other lamellar structures.

The plan of the paper is as follows: in Section 2 the main equations of the model are presented and how the PST morphology affects the critical stresses and the constitutive behavior inside each lamella are discussed. In Section 3 the predicted PST anisotropy is validated by comparison with the present experiments and also with classical results found in the literature. In Section 4 some advantages, limitations, possible improvements and further applications of this basic model of PST plasticity are addressed.

\section{MODEL}

The morphology of PST crystals influences the mechanical behavior of the $\gamma$-TiAl phase in various ways. Thus, the plastic behavior of an isolated $\gamma$ TiAl single crystal is first described, then how the morphology of a PST crystal can affect the critical stresses of slip and twinning systems is discussed and finally two of these single crystals as constituent lamellae (i.e. matrix and twin) of a PST are considered in order to account for the effect of morphology on the constitutive behavior of the lamellar structure as a whole. Although this assumption of a 2-lamellae structure is a simplification of the actual six-domain structure (Fig. 1), it will be shown that it allows the main effect of morphology on the PST behavior to be captured.

\subsection{Constitutive behavior of a $\gamma$-TiAl single crystal}

According to Mecking et al. [22], the active deformation modes in $\gamma$-TiAl are: $\{111\}\langle 110]$ slip by ordinary dislocations, $\{111\}<101]$ slip by super dislocations and $\{111\}\langle 11 \overline{2}]$ twinning (Table 1). These deformation modes have been confirmed to be the most active ones in PST crystals by Kishida et al. [23]. In the present formulation, twinning will be treated as a deformation mode with an assigned critical resolved shear stress like the other slip modes. Unlike slip, twinning is not operative in the opposite sense of the twinning vector because this would require an extremely high shear stress.

When a stress is acting upon a single crystal, the shear rate on a given slip system $(s): \dot{\gamma}^{s}$ can be

Table 1. Deformation systems of $\gamma$-TiAl and their "mechanismbased" and "morphology-based" classification

\begin{tabular}{lcc}
\hline System & $\begin{array}{c}\text { Mechanism-based } \\
\text { classification (modes) }\end{array}$ & $\begin{array}{c}\text { Morphology-based } \\
\text { classification (types) }\end{array}$ \\
\hline$(1 \overline{1} 1)[110]$ & ordinary slip & transversal slip \\
$(\overline{1} 11)[110]$ & ordinary slip & transversal slip \\
$(111)[1 \overline{1} 0]$ & ordinary slip & mixed slip \\
$(\overline{11} 1)[1 \overline{1} 0]$ & ordinary slip & longitudinal slip \\
$(\overline{1} 11)[0 \overline{1} 1]$ & super slip & transversal slip \\
$(1 \overline{1} 1)[\overline{1} 01]$ & super slip & transversal slip \\
$(111)[0 \overline{1} 1]$ & super slip & transversal slip \\
$(111)[\overline{1} 01]$ & super slip & transversal slip \\
$(1 \overline{1})[011]$ & super slip & mixed slip \\
$(\overline{1} 11)[101]$ & super slip & mixed slip \\
$(\overline{11} 1)[011]$ & super slip & longitudinal slip \\
$(\overline{11} 1)[101]$ & super slip & longitudinal slip \\
$(\overline{1} 11)[\overline{1} 1 \overline{2}]$ & twinning & transversal twinning \\
$(1 \overline{1} 1)[1 \overline{12}]$ & twinning & transversal twinning \\
$(111)[11 \overline{2}]$ & twinning & transversal twinning \\
$(\overline{11} 1)[\overline{11}]$ & twinning & longitudinal twinning \\
\hline
\end{tabular}


expressed-for constant temperature and thermally activated slip-by means of the rate-sensitivity approach, i.e. a microscopic equivalent of the Norton law for creep:

$$
\dot{\gamma}^{s}=\dot{\gamma}_{0}\left(\frac{\tau_{\mathrm{r}}^{s}}{\tau_{0}^{s}}\right)^{n}=\dot{\gamma}_{0}\left(\frac{m_{i j}^{s} \sigma_{i j}{ }^{\prime}}{\tau_{0}^{s}}\right)^{n}
$$

where $\tau_{\mathrm{r}}^{s}$ and $\tau_{0}^{s}$ are the resolved and critical stress of system $(s)$, respectively; $m_{i j}^{s}$ is the Schmid tensor of system $(s) ; \sigma_{i j}{ }^{\prime}$ is the deviatoric stress; $\dot{\gamma}_{0}$ is a reference strain rate and $n$ is the inverse of the rate sensitivity. Then, if a given strain rate is applied to a single crystal, its response can be obtained as

$$
\dot{\varepsilon}_{i}=\sum_{s} m_{i}^{s} \dot{\gamma}^{s}=\dot{\gamma}_{0} \sum_{s} m_{i}^{s}\left(\frac{m_{j}^{s} \sigma_{j}^{\prime}}{\tau_{0}^{s}}\right)^{n} \quad(i, j=1,5) .
$$

In equation (2) there is an implicit summation over the repeated index $j$ and the traceless tensors are expressed as 5-dim vectors using a modified Lequeu [24] convention (i.e. with the third and fourth components interchanged):

$$
\begin{aligned}
& T_{1}=\frac{1}{\sqrt{2}}\left(T_{22}-T_{11}\right), T_{2}=\sqrt{\frac{3}{2}} T_{33}, \\
& T_{3}=\sqrt{2} T_{13}, T_{4}=\sqrt{2} T_{23}, T_{5}=\sqrt{2} T_{12}
\end{aligned}
$$

where $T_{i j}$ and $T_{k}$ are a generic traceless tensor and its vectorial representation, respectively. From equation (2) the stress can be calculated by solving a $5 \times 5$ system of non-linear equations for each single crystal. As in equation (2), in the following equations indexes $i$ and $j$ will vary from 1 to 5 , unless they are explicitly quoted.

\subsection{Critical stresses}

In an isolated $\gamma$-TiAl single crystal, prior to any deformation, the critical stresses $\tau_{0}^{s}$ of every deformation system of the same mode (i.e. ordinary slip, super slip and twinning) should be the same but they can vary from mode to mode (e.g. the critical stresses of ordinary slip can be different from super slip, etc.). This distinction between ordinary and super slip and twinning will be referred to in what follows as mechanism-based classification of critical stresses (Table 1, second column).

Now consider a simplified lamellar PST structure, formed by one matrix and one twin, having the matrix and the twin lamellae the orientations of domains $\mathrm{I}^{\mathrm{M}}$ and $\mathrm{I}^{\mathrm{T}}$ in Fig. 1, respectively. This matrix-twin pair corresponds to a (111)[112] twinning system and therefore a reference frame (called "lamellar axes" in what follows) is adopted having axis $x_{3}^{\mathrm{L}}$ lying along the twinning direction [112], axis $x_{2}^{\mathrm{L}}$ along [111] (i.e. normal to the twinning plane) and axis $x_{1}^{\mathrm{L}}$ along [1̄ㅣㄹ (see Fig. 1).

When a single crystal is a part of this structure, it can be rationally assumed that the morphology determines differences between the critical stresses of deformation systems of the same mode, at very early stages of deformation, i.e. the critical stress may be different for systems of the same slip or twinning mode depending on the orientation of each system with respect to the lamellar plane. This feature allows a new classification of deformation systems, i.e. the morphology-based classification (Table 1, third column). For instance, it is possible to find (in both lamellae) one ordinary slip system, two super slip systems and one twinning

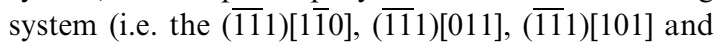
(111)[112] systems, respectively) which have their slip (or twinning) direction and slip (or twinning) plane parallel to the interface. In this new morphology-based classification these slip (or twinning) systems will be grouped in the longitudinal slip (or twinning) type of deformation. Furthermore, each lamella contains one ordinary slip system and two super slip systems with their slip direction parallel to the interface and with the slip plane transversal to the interface (i.e. (111)[1̄ㅣ, (111)[101] and (111)[011]). These slip systems will be grouped in the mixed slip type. Finally, the rest of the ordinary, super and twinning systems have their slip (or twinning) directions and their slip (or twinning) planes transversal to the interface. These slip (or twinning) systems will belong to transversal slip (or twinning) type.

The Hall-Petch effect has been actually confirmed in equiaxed microstructures of $\gamma$-TiAl alloys [2,25] and also by Umakoshi and Nakano in PST crystals deformed under compression for different orientations [26]. This allows a qualitative analysis of the expected relative critical stresses of the different morphology-based types of deformation. Due to the high aspect ratio of the lamellae of PST crystals different "effective" grain sizes for systems of the longitudinal and the transversal types of deformation should be considered in the Hall-Petch law. When the lamellae are perpendicular to the loading axis, the yield stress can be correlated with the lamellar spacing, i.e. the short axis of the lamellae. In this case, the deformation is carried out mainly by systems of the transverse type which should have high critical stresses. When the lamellae form an angle of $45^{\circ}$ with the compression axis, the yield stress can be correlated with the domain size, i.e. the long axis of the lamellae. It follows that the active deformation systems are of the longitudinal type and their critical stresses are low. Finally, when the lamellae are parallel to the compression axis the systems of the mixed slip type are the most active. In terms of slip geometry, it is expected in this case that the yield stresses were controlled by the domain size, as well. Nevertheless, Umakoshi and Nakano found that the lamellar spacing also has a strong influence on the critical stress of slip systems of the mixed type [26]. Therefore, an intermediate yield stress is expected for these mixed type systems. 
The reason why the simplified 2-lamellae structure is able to capture the main effect of morphology on the PST behavior relies on this morphology-based classification. The latter provides a rational way to average over the whole set of deformation systems, within all the six domains of a PST, to give a good representation of the overall behavior of a PST crystal. In fact, due to the crystallographic arrangement of the domains, a PST crystal should deform activating all deformation modes simultaneously. This becomes obvious in the case of slip (or twinning) of the longitudinal type propagating along a lamella from one domain into another. In this case, due to the $120^{\circ}$ rotation of the domains around the normal direction of the lamella, a dislocation must switch from an ordinary one to a super dislocation (and vice versa) several times and, in some domains, a combination of ordinary slip and twinning is favorable instead of super slip. A detailed analysis of this has been given by Kishida et al. [23].

\subsection{Constitutive behavior of a lamellar structure}

1. Consider a strain rate $\overline{\dot{\varepsilon}}_{i}$ applied to the matrixtwin structure sketched in Fig. 1. If labels $\mathrm{M}$ and $\mathrm{T}$ identify the matrix and twin, respectively, the constitutive relation of the ensemble - in lamellar axes - can be written as

$$
\begin{aligned}
\dot{\dot{\varepsilon}}_{i}=\dot{\gamma}_{0}\left(w^{\mathrm{M}} \sum_{s} m_{i}^{s, \mathrm{M}}\left(\frac{m_{j}^{s, \mathrm{M}} \sigma_{j}{ }^{\prime \mathrm{M}}}{\tau_{0}^{s, \mathrm{M}}}\right)^{n}\right. \\
\left.+w^{\mathrm{T}} \sum_{s} m_{i}^{s, \mathrm{~T}}\left(\frac{m_{j}^{s, \mathrm{~T}} \sigma_{j}{ }^{\prime \mathrm{T}}}{\tau_{0}^{s, \mathrm{~T}}}\right)^{n}\right) \\
\dot{\varepsilon}_{1}^{\mathrm{M}}=\dot{\varepsilon}_{1}^{\mathrm{T}} \\
\dot{\varepsilon}_{2}^{\mathrm{M}}=\dot{\varepsilon}_{2}^{\mathrm{T}} \\
\dot{\varepsilon}_{3}^{\mathrm{M}}=\dot{\varepsilon}_{3}^{\mathrm{T}} \\
\sigma_{4}{ }^{\prime \mathrm{M}}=\sigma_{4}{ }^{\prime \mathrm{T}} \\
\sigma_{5}{ }^{\prime \mathrm{M}}=\sigma_{5}{ }^{\prime \mathrm{T}}
\end{aligned}
$$

where $\left(\dot{\varepsilon}^{\mathrm{M}}, \sigma^{\prime \mathrm{M}}\right)$ and $\left(\dot{\varepsilon}^{\mathrm{T}}, \sigma^{\prime \mathrm{T}}\right)$ are the local states inside the matrix and the twin; $s, \mathrm{M}$ and $s, \mathrm{~T}$ identify the slip and twinning systems in both lamellae and $w^{\mathrm{M}}$ and $w^{\mathrm{T}}$ are weight factors proportional to the relative volumes of matrix and twin, respectively. In writing equation (4a) it has been assumed that the overall strain rate is given by a weighted average of the strain rate inside each lamella. Moreover, equations (4b)-(f) are based on the RC concept [16]. According to the $\mathrm{RC}$ theory, since the lamellae are flat (being the short direction along $x_{2}^{\mathrm{L}}$ ) the only local strainrate components that are allowed to be different are shear rates $\dot{\varepsilon}_{4}$ and $\dot{\varepsilon}_{5}\left(\dot{\varepsilon}_{23}\right.$ and $\dot{\varepsilon}_{12}$, respectively). This follows from the fact that the differences in those shears between both lamellae can be accommodated without large spatial incompatibilities. Correspondingly, the continuity across the interface of the other three strain-rate components and of the fourth and fifth components of the stress should be enforced. Moreover, equations (4e)-(f) are two (out of three) necessary conditions for equilibrium of forces across the interface. The third condition, i.e. the continuity of the third component of the Cauchy stress (i.e. $\sigma_{22}^{\mathrm{T}}=\sigma_{22}^{\mathrm{M}}$ ) can be also fulfilled by imposing adequate hydrostatic states inside each lamella. Equations (4) give a $10 \times 10$ nonlinear system of equations where the unknowns are the five components of stress inside each layer. The overall stress in the structure is obtained as

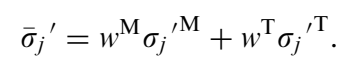

System (4) can be extended to the general case of a stack formed by $N$ lamellae. Each lamella can have different crystallographic orientation and volume but they should be parallel to each other. The constitutive behavior of such a structure can be obtained from

$$
\begin{gathered}
\overline{\dot{\varepsilon}}_{i}=\dot{\gamma}_{0} \sum_{K=\mathrm{I}}^{N} w^{K} \sum_{s} m_{i}^{s, K}\left(\frac{m_{j}^{s, K} \sigma_{j}{ }^{\prime K}}{\tau_{0}^{s, K}}\right)^{n} \\
\dot{\varepsilon}_{1}^{\mathrm{I}}=\dot{\varepsilon}_{1}^{\mathrm{II}}=\ldots=\dot{\varepsilon}_{1}^{N} \\
\dot{\varepsilon}_{2}^{\mathrm{I}}=\dot{\varepsilon}_{2}{ }^{\mathrm{II}}=\ldots=\dot{\varepsilon}_{2}{ }^{N} \\
\dot{\varepsilon}_{3}^{\mathrm{I}}=\dot{\varepsilon}_{3}{ }^{\mathrm{II}}=\ldots=\dot{\varepsilon}_{3}{ }^{N} \\
\sigma_{4}{ }^{\mathrm{I}}=\sigma_{4}{ }^{\prime \mathrm{II}}=\ldots=\sigma_{4}{ }^{\prime N} \\
\sigma_{5}{ }^{\mathrm{I}}=\sigma_{5}{ }^{\prime \mathrm{II}}=\ldots=\sigma_{5}{ }^{\prime N}
\end{gathered}
$$

where the indexes $K=\mathrm{I}, \mathrm{II}, \ldots, N$ identify the different lamellae and $\left(\dot{\varepsilon}^{K}, \sigma^{\prime K}\right), s, K$ and $w^{K}$ are the local state, the slip and twinning systems and the weight factor of lamella $K$, respectively. System (6) consists of $5 \times K$ unknowns, i.e. the five components of the local stress in each layer and $5 \times K$ equations, i.e. five from equation (6a) and $5 \times(K-1)$ from equations (6b)-(f). The overall stress in the structure is

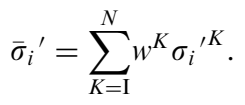

The general case of $N$ lamellae is not analyzed in detail here. The latter equations can be eventually used as an extension of the present model for more complicated lamellar structures consisting of a stack of more than two lamellae. 
Nevertheless, equations (6) and (7) should not be confused with the general solution for the sixdomain PST structure. In the latter, sets of three domains are adjacent within each lamella, i.e. they do not form a 6-lamellae stack.

2. Consider there is a stress $\bar{\sigma}_{i}{ }^{\prime}$ (instead of a strain rate) applied to the matrix-twin structure. Using similar considerations as in the former case based on the RC theory, the set of equations that describes the mechanical behavior of such a structure is given by

$$
\begin{aligned}
& \bar{\sigma}_{i}{ }^{\prime}=w^{\mathrm{M}}{\sigma_{i}}^{\prime} \mathrm{M}+w^{\mathrm{T}}{\sigma_{i}}^{\prime}{ }^{\mathrm{T}} \quad(i=1,3) \\
& \dot{\gamma}_{0} \sum_{s} m_{i}^{s, \mathrm{M}}\left(\frac{m_{j}^{s, \mathrm{M}}{\sigma_{j}}^{\prime \mathrm{M}}}{\tau_{0}^{s, \mathrm{M}}}\right)^{n}= \\
& \dot{\gamma}_{0} \sum_{s} m_{i}^{s, \mathrm{~T}}\left(\frac{m_{j}^{s, \mathrm{~T}}{\sigma_{j}}{ }^{\mathrm{T}}}{\tau_{0}^{s, \mathrm{~T}}}\right)^{n} \quad(i=1,3) \\
& \sigma_{4}{ }^{\prime \mathrm{M}}={\sigma_{4}}{ }^{\mathrm{T}}=\bar{\sigma}_{4}{ }^{\prime} \\
& \sigma_{5}{ }^{\mathrm{M}}={\sigma_{5}}{ }^{\prime \mathrm{T}}=\bar{\sigma}_{5}{ }^{\prime} .
\end{aligned}
$$

Formally, the set of equations (8) is also a $10 \times 10$ non-linear system, the unknowns being the five stress components inside each lamella. Nevertheless, the fourth and fifth components in each lamella can be trivially obtained from equations (8c)-(d) and therefore a $6 \times 6$ system should be actually solved. Once the stresses are known, the overall strain rate can be obtained as

$$
\begin{aligned}
\overline{\dot{\varepsilon}}_{i}=\dot{\gamma}_{0}\left(w^{\mathrm{M}} \sum_{s} m_{i}^{s, \mathrm{M}}\left(\frac{m_{j}^{s, \mathrm{M}} \sigma_{j}{ }^{\prime \mathrm{M}}}{\tau_{0}^{s, \mathrm{M}}}\right)^{n}\right. \\
\left.+w^{\mathrm{T}} \sum_{s} m_{i}^{s, \mathrm{~T}}\left(\frac{m_{j}^{s, \mathrm{~T}} \sigma_{j}{ }^{\prime \mathrm{T}}}{\tau_{0}^{s, \mathrm{~T}}}\right)^{n}\right) .
\end{aligned}
$$

Finally, if a stress state is applied to an $N$-lamellae structure, the system to be solved to get its mechanical response is

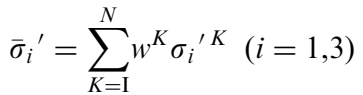

$$
\begin{aligned}
& \dot{\varepsilon}_{1}^{\mathrm{I}}=\dot{\varepsilon}_{1}^{\mathrm{II}}=\ldots=\dot{\varepsilon}_{1}^{N} \\
& \dot{\varepsilon}_{2}^{\mathrm{I}}=\dot{\varepsilon}_{2}^{\mathrm{II}}=\ldots=\dot{\varepsilon}_{2}^{N} \\
& \dot{\varepsilon}_{3}^{\mathrm{I}}=\dot{\varepsilon}_{3}^{\mathrm{II}}=\ldots=\dot{\varepsilon}_{3}^{N} \\
& {\sigma_{4}}^{\prime I}={\sigma_{4}}{ }^{\text {II }}=\ldots={\sigma_{4}}{ }^{N}=\bar{\sigma}_{4}{ }^{\prime} \\
& \sigma_{5}{ }^{\mathrm{I}}=\sigma_{5}{ }^{\prime \mathrm{II}}=\ldots=\sigma_{5}{ }^{\prime N}=\bar{\sigma}_{5}{ }^{\prime}
\end{aligned}
$$

and the overall strain rate is given by

$$
\overline{\dot{\varepsilon}}_{i}=\dot{\gamma}_{0} \sum_{K=\mathrm{I}}^{N} w^{K} \sum_{s} m_{i}^{s, K}\left(\frac{m_{j}^{s, K} \sigma_{j}{ }^{\prime K}}{\tau_{0}^{s, K}}\right)^{n} .
$$

\section{RESULTS AND VALIDATION}

\subsection{Experimental evidence}

In this section the model will be validated by comparison with two sets of compression tests done on $\gamma$-TiAl-based PST crystals and as-cast material where the angles between the lamellar boundary and the loading axis were varied. These are: (a) the classical Yamaguchi yield stress results for PST crystals of composition $\mathrm{Ti}-49.3$ at.\% $\mathrm{Al}$ [13] and (b) measurements of the transversal strains after compressing as-cast material with strongly oriented lamellae and a composition of $\mathrm{Ti}-48$ at. $\%$ Al2 at. $\% \mathrm{Cr}$.

The yield stress of a PST crystal deformed in uniaxial compression is high when the lamellae are parallel or perpendicular to the loading axis and low for intermediate orientations. Figure 2 shows the dependence of the yield stress with the angle between the lamellae and the loading axis. In what follows, the angle $\phi$ varies due to a rotation of the crystal around a $\langle 110\rangle$ direction, as sketched in Fig. 3. The "sample axes" reference frame-defined by axis $x_{3}^{\mathrm{S}}$ parallel to the loading direction, axis $x_{1}^{\mathrm{S}}$ along the rotation axis and axis $x_{2}^{\mathrm{S}}$ perpendicular to the former ones-should not be confused with the set of "lamellar axes" defined earlier to formulate the constitutive equation of the lamellar structure (in fact, the "lamellar axes" and the "sample axes" coincide for $\phi=0$ ). A similar profile is obtained in the case of uniaxial tension or when the angle $\phi$ is varied by rotation around a $\langle 112\rangle$ direction [13]. The orientation of the lamellar boundary with respect to the (vertical) loading axis is sketched on top of each experimental point.

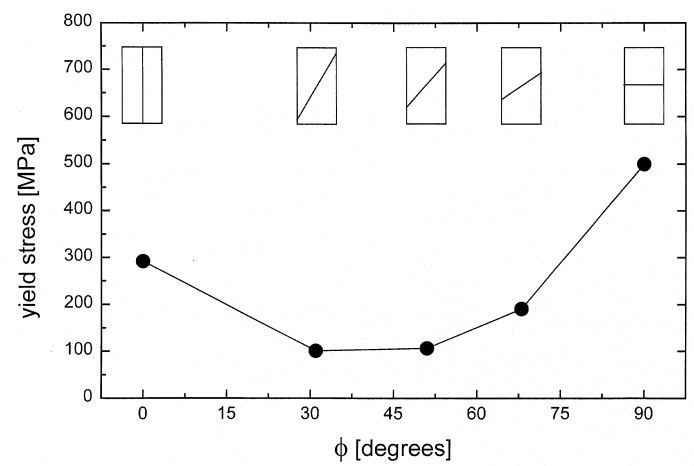

Fig. 2. Experimental yield stresses of PST crystals (Ti49.3 at. \% Al) in uniaxial compression for different angles $\phi$ (i.e. the angle between the lamellae and the compression axis). $\phi$ varies by rotation around a $\langle 110\rangle$ direction lying in the lamellar plane [13] (see also Fig. 3). 


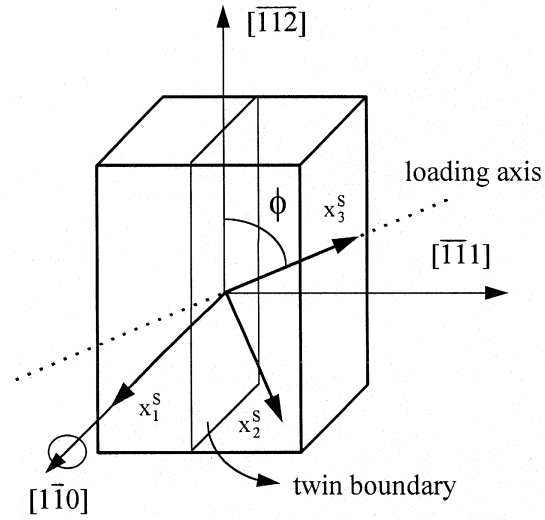

Fig. 3. Definition of the "sample axes" reference frame. $x_{3}^{\mathrm{S}}$ is parallel to the loading axis, $x_{1}^{\mathrm{S}}$ is the rotation axis, lying along a $\langle 110\rangle$ direction in the lamellar plane and $x_{2}^{\mathrm{S}}$ is perpendicular to $x_{1}^{\mathrm{S}}$ and $x_{3}^{\mathrm{S}}$.

The transversal strains of the as-cast material, measured after uniaxial compression, also exhibit a strong dependence with angle $\phi$. Due to the polycrystalline microstructure of the as-cast material, $\phi$ is defined in this case as the mean angle between the lamellar planes and the compression axis. Furthermore, unlike in the case of PST crystals, no distinction can be made between a rotation around a $\langle 110\rangle$ or a $\langle 112\rangle$ direction in order to vary $\phi$ because the material has a $\langle 111\rangle$-fiber texture with the fiber axis being the normal of the lamellae. During the solidification of the cast material, lamellar dendrites formed. As the mismatch between the orientation of the lamellae is very small the material exhibits an anisotropic behavior which is very similar to that of a single set of lamellae (i.e. a PST) [10].

Figure 4 shows the variation of the ratios of the transversal strains and the compression strain (i.e. $\varepsilon_{11} / \varepsilon_{33}$ and $\varepsilon_{22} / \varepsilon_{33}$, in sample axes) for different angles $\phi$, after uniaxial compression tests. The mean orientation of the lamellar boundaries with respect to the (vertical) loading axis is sketched on top of the experimental points. When the lamellae are parallel to the loading axis $(\phi=0)$ the strain is such that the component perpendicular to the lamellar boundary (i.e. $\varepsilon_{22}$ for $\phi=0$ ) is small and most of the transversal strain is carried along the lamellar planes. As soon as the loading axis is inclined, the trend changes drastically and the strain component along the $\varepsilon_{11}$ direction becomes smaller than $\varepsilon_{22}$. Finally, at $\phi=90$, when the loading axis is normal to the lamellae, the test becomes axially symmetric and therefore both transversal strains are almost equal.

\subsection{Model assessment}

Unlike a rigid-plastic material, it is not possible to get true yield points (and therefore a true yield

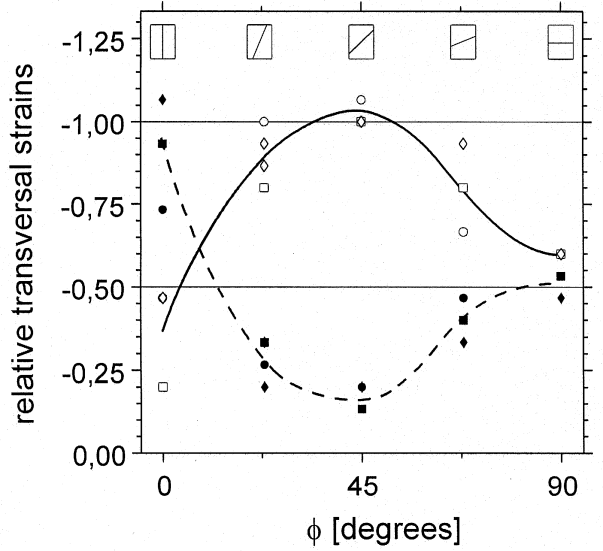

Fig. 4. Transversal strains measured after uniaxial compression on as-cast material (Ti-48 at.\% Al-2 at.\% $\mathrm{Cr}$ ) with strongly oriented lamellae, for different angles $\phi$ (i.e. angle between the compression axis and the mean direction of the lamellar plane). Transversal strains are normalized to the compression strain. Black and white symbols show the values for deformation along $x_{1}^{\mathrm{S}}\left(\varepsilon_{11}\right)$ and $x_{2}^{\mathrm{S}}\left(\varepsilon_{22}\right)$, respectively.

surface) of a viscoplastic material. Nevertheless, the relative anisotropy of a viscoplastic material can be estimated by comparing points that belong to the same equipotential surface. This requires to prove the single crystal or the lamellar structure in a given stress direction and then to correct the stress modulus in order to be exactly at the reference potential, using the procedure described in Appendix A. In the limit, when the rate sensitivity of the viscoplastic material tends to zero, the shape of this equipotential surface tends to the shape of the yield surface of the rate-insensitive material. In what follows, a high exponent $n$ (i.e. $n=19$ ) will be used to calculate approximate values of relative yield stresses for a PST crystal loaded in different directions.

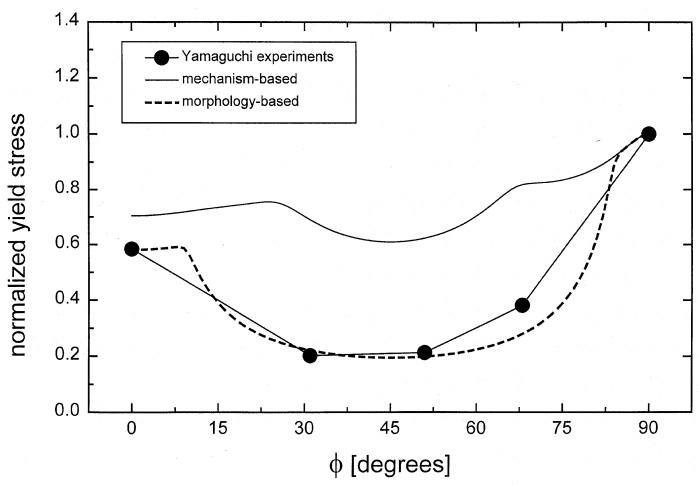

Fig. 5. Comparison of experimental yield stresses (black circles) with model predictions for mechanism-based (solid line) and optimized morphology-based critical stresses (dash line). All data are normalized to the yield stress at $\phi=90^{\circ}$. 
The set of equations (8) (i.e. stress applied to a matrix-twin structure) has been solved for different directions of an applied uniaxial compression stress in order to test the model with respect to the experimental curves shown in Figs 2 and 4. The calculated results for the yield stresses and macroscopic transversal deformations are shown in Figs 5 and 6, respectively. For comparison of the calculated relative yield stresses and the experimental ones, a normalization with respect to the value at $\phi=90^{\circ}$ was performed in Fig. 5. This implies that both values, calculated and experimental, are equal at $\phi=90^{\circ}$ in every case.

In a first attempt the critical stresses for all deformation modes were set equal $\left(\tau_{0}^{\text {ord }}=\tau_{0}^{\text {sup }}=\tau_{0}^{\text {tw }}=1\right)$ which is the simplest choice of critical stresses based on the mechanism-based classification. This simple case already reproduces in a qualitative way the trend of high yield stress at $\phi=0^{\circ}$ and $90^{\circ}$ as well as low yield stress at $\phi=45^{\circ}$. Nevertheless, the calculated relative values of yield stress do not match quantitatively with the experimental data. Concerning the predictions of the transversal strains, the general trend of the experimental curves in Fig. 4 is well reproduced but the turnover from plain strain in the $x-z$ plane to plain strain in the $y-z$ plane appears at an angle of $\phi \approx 25^{\circ}$ while in the experiments this happens at a smaller angle. However, a very detailed comparison is difficult because, as already quoted in Section 3.1, the experiments were done with as-cast material which in comparison with a PST is expected to exhibit less significant changes in yield stress [10] and transversal strains.

A better quantitative matching between the predicted anisotropy and the experimental evidence can be obtained using the morphology-based classi- fication described in Section 2.2. The morphologybased classification of deformation systems provides two parameters which can be used to optimize the model to match with the experimental data. These parameters are the ratios between the critical stresses of transversal, mixed and longitudinal types of deformation, i.e. $\tau_{0}^{\text {trans }}: \tau_{0}^{\text {long }}$ and $\tau_{0}^{\text {mix }}: \tau_{0}^{\text {long }}$ where $\tau_{0}^{\text {trans }}$ is the critical stress assigned to both the slip and twinning transversal types (assumed equal for simplicity) and $\tau_{0}^{\text {long }}$ is the critical stress assigned to both the slip and twinning longitudinal types. However, in this fitting procedure, the values of the critical stresses should not be arbitrary but they must be consistent with the Hall-Petch arguments discussed in Section 2.2, which in the present notation, can be expressed as: $\tau_{0}^{\text {long }}<\tau_{0}^{\text {mix }}<\tau_{0}^{\text {trans }}$.

As mentioned in Section 2.2, at $\phi=90^{\circ}$ the deformation is carried out by systems of the transverse type, at intermediate orientations (around $\phi=45^{\circ}$ ) by systems of the longitudinal type and for $\phi=0^{\circ}$ by systems of the mixed type. This has been also confirmed by simulations. Table 2 shows deformation systems which are active in the matrix and the twin lamellae. The same pattern of active systems is obtained for the considered range of critical stresses. Therefore, the ratios $\tau_{0}^{\text {trans }}: \tau_{0}^{\text {long }}$ and $\tau_{0}^{\text {mix }}: \tau_{0}^{\text {long }}$ can be used separately to optimize the calculated yield stresses for the best matching with the measured values at $\phi=0^{\circ}$ and $45^{\circ}$, respectively. By performing this optimization procedure, it was found that the values $\tau_{0}^{\text {trans }}: \tau_{0}^{\text {long }}=3.33$ and $\tau_{0}^{\text {mix }}: \tau_{0}^{\text {long }}=2.72$ are the best choice (see Fig. 5). With these parameters, the calculated transversal strains show a turnover from plain strain in the $x-z$ plane to plain strain in the $y-z$ plane at an angle $\phi$ between $9^{\circ}$ and $10^{\circ}$ (see Fig. 6) which is closer to the experimental observations in as-cast material. a) mechanism-based

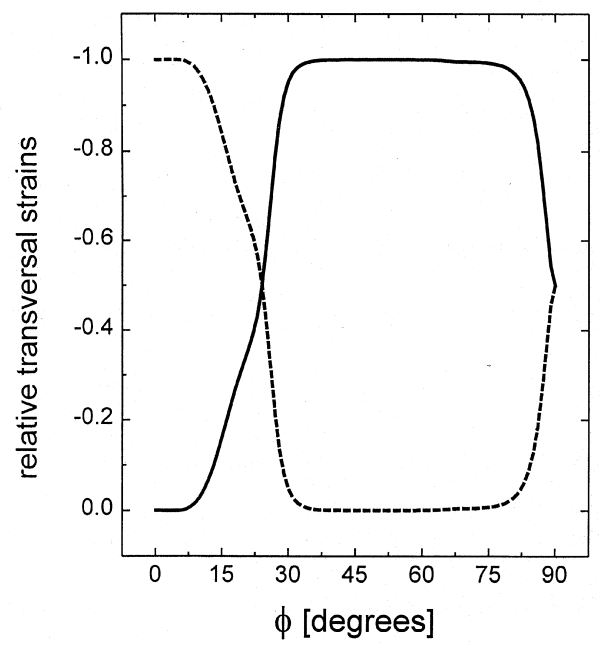

b) morphology-based

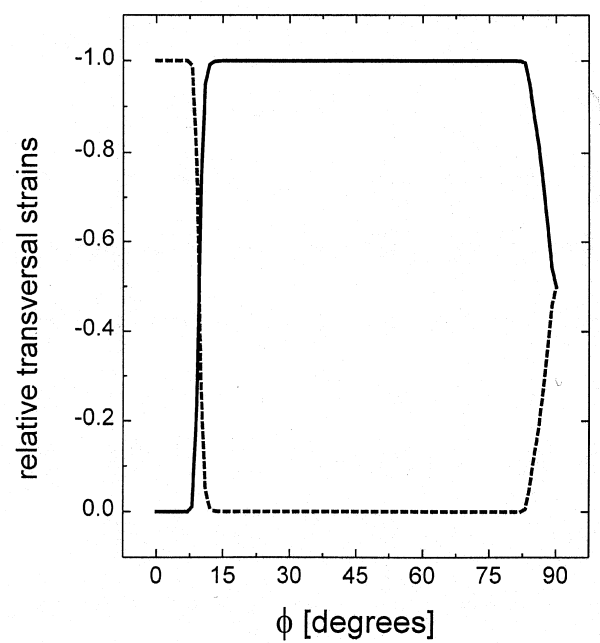

Fig. 6. Calculated transversal strains for uniaxial compression along $x_{1}^{\mathrm{S}}$ (dash line) and $x_{2}^{\mathrm{S}}$ (solid line) (a) mechanism-based and (b) optimized morphology-based classification of critical stresses. 
Table 2. Calculated activity of deformation systems under compression in matrix and twin, respectively and their morphology-based classification, for different angles $\phi$. No change of this pattern has been observed in the considered range of critical stresses

\begin{tabular}{|c|c|c|c|c|}
\hline \multirow{2}{*}{$\phi$} & \multicolumn{3}{|c|}{ Active systems } & \multirow{2}{*}{$\begin{array}{l}\text { Morphology- } \\
\text { based } \\
\text { classification }\end{array}$} \\
\hline & Matrix & & Twin & \\
\hline $0^{\circ}$ & & $\begin{array}{l}(1 \overline{1} 1)[011] \\
(\overline{1} 11)[101]\end{array}$ & & mixed slip \\
\hline $45^{\circ}$ & $\begin{array}{l}(\overline{11} 1)[011] \\
(\overline{11} 1)[011]\end{array}$ & & $(\overline{11} 1)[\overline{112}]$ & $\begin{array}{l}\text { longitudinal slip/ } \\
\text { longitudinal } \\
\text { twinning }\end{array}$ \\
\hline $90^{\circ}$ & & $\begin{array}{l}(1 \overline{1} 1)[110] \\
(\overline{1} 11)[110] \\
(\overline{1} 11)[0 \overline{1} 1] \\
(1 \overline{1} 1)[\overline{1} 01] \\
(111)[0 \overline{1} 1] \\
(111)[\overline{1} 01]\end{array}$ & & transversal slip \\
\hline
\end{tabular}

\section{CONCLUSIONS}

A crystal plasticity model has been developed which is able to describe the response of a multilayered, lamellar crystal structure under any applied multiaxial stress or strain rate. The model has been applied to a $\gamma$-TiAl-based PST crystal and it has been validated by comparison with experimental results.

The PST crystal has been reduced to one matrixtwin pair of crystals. According to the model, the plastic response of two joined, twinned crystals with a high aspect ratio can be calculated from a set of equations based on: (1) a rate-sensitive approach to describe the single crystal behavior and (2) the relaxed constraints theory to account for the influence of the morphology during the co-deformation of both lamellae. A morphology-based classification for the assignment of critical stresses of the deformation systems has been introduced. The physical argument behind this classification is based on the extension of the Hall-Petch effect to each lamella, i.e. the critical stresses are determined by the "effective grain size" associated with each individual deformation system (small for transversal and large for longitudinal dislocation movement) rather than by the deformation mode (ordinary or super slip and twinning). Based on this morphology-based classification, two parameters were introduced to describe the relative ease of longitudinal, mixed and transversal slip. These parameters were fitted to match the predicted anisotropy with data obtained from uniaxial compression tests.

Calculations based on crystal plasticity models allow the yield surface of single crystals as well as of textured polycrystals to be described. As a natural extension, the present model of PST plasticity can be used to calculate the yield surface of a PST crystal. This means that it will be possible to predict the PST response under any applied strain and/or stress state, i.e. information which cannot be easily accessed by experiments because these are usually limited to uniaxial tests or other simple geometries. Examples of these PST yield surface predictions can be found elsewhere [27].

The model, as presented here, has some simplifications, i.e. the PST is assumed to be formed by two twin lamellae instead of by the complete set of six crystallographically related domains. Therefore, although the overall behavior of a PST is well described by this two-lamella model, the present approach is not able to describe properly the activity of the slip systems at a microscopic scale. For a detailed analysis of the microscopic mechanisms inside each domain of a PST crystal, the present model is currently being extended to solve the sixdomain problem.

Among other potential applications of the present approach, two attractive possibilities are:

1. The PST plasticity model can be implemented as the local constitutive relation within a Taylor model or one-site VPSC code. This will allow the prediction of the plastic response and the texture development of a textured lamellar polycrystal. This kind of model can be thought of as "2-scale models", i.e. relaxed constraints at the local (PST) level and Taylor or VPSC at the macroscopic level.

2. The present model can be also used within a crystal plasticity-based finite elements (FE) code. For this purpose, the yield surface of a PST can be calculated to be used by a FE code to investigate, for instance, the stress and strain inhomogeneities at the scale of lamellar colonies.

Acknowledgements-The support of the DFG, within the scope of the Sonderforschungsbereich 371, is gratefully acknowledged. 


\section{REFERENCES}

1. Clemens, H., Glatz, W., Schretter, P., Koeppe, C., Bartels, A., Behr, R. and Wanner, A., in Gamma Titanium Aluminides, ed. Y.-W. Kim, R. Wagner and M. Yamaguchi. The Minerals, Metals and Materials Society, 1995, p. 717.

2. Koeppe, C., Bartels, A., Clemens, H., Schretter, P. and Glatz, W., Mater. Sci. Engng A, 1995, 201, 182.

3. Kim, Y.-W., J. Mater. Sci., 1994, No. 6, 30.

4. Bartels, A., Koeppe, C. and Mecking, H., Mater. Sci. Engng A, 1995, 192/193, 226.

5. Bartels, A., Hartig, Ch., Willems, St. and Uhlenhut, H., Mater. Sci. Engng A, 1997, 239/240, 14.

6. Es-Souni, M., Bartels, A. and Wagner, R., in Structural Intermetallics, ed. R. Darolia, J. J. Lewandowski, C. T. Liu, P. L. Martin, D. B. Miracle and M. V. Nathal. The Minerals, Metals and Materials Society, 1993, p. 335.

7. Es-Souni, M., Bartels, A. and Wagner, R., Mater. Sci. Engng A, 1993, 171, 127.

8. Es-Souni, M., Bartels, A. and Wagner, R., Acta metall. mater., 1995, 43, 153

9. Seeger, J., Hartig, Ch., Bartels, A. and Mecking, H., Mater. Res. Soc. Symp. Proc., 1991, 213, 157.

10. Uhlenhut, H., Willems, St., Bartels, A. and Mecking, H., Proceedings of the 4th European Conference on Advanced Materials and Processes, EuroMat 95, Padua/Venice, Italy, 25-28 September 1995, Symposium G, p. 49.

11. Johnson, D. R., Masuda, Y., Inui, H. and Yamaguchi, M., Acta mater., 1997, 45, 2523.

12. Fujiwara, T., Nakamura, A., Hosomi, M., Nishitani, S. R., Shirai, Y. and Yamaguchi, M., Phil. Mag. A, 1990, 61, 591.

13. Yamaguchi, M., ISIJ Int., 1991, 31(10), 1127.

14. Inui, H., Oh, M. H., Nakamura, A. and Yamaguchi, M., Phil. Mag. A, 1992, 539.

15. Taylor, G. I., J. Inst. Metals, 1938, 62, 307.

16. Honneff, H. and Mecking, H., in Proc. ICOTOM-6, ed. S. Nagashima. The Iron and Steel Inst. of Japan, Tokyo, 1981, p. 347.

17. Molinari, A., Canova, G. R. and Ahzi, S., Acta metall., 1987, 35, 2983.

18. Lebensohn, R. A. and Tomé, C. N., Acta metall. mater., 1993, 41, 2611.

19. Tomé, C. N., Lebensohn, R. A. and Kocks, U. F., Acta metall., 1991, 39, 2667.

20. Lebensohn, R. A., Wenk, H.-R. and Tomé, C. N., Acta mater., 1998, 46, 2683.

21. Lebensohn, R. A. and Canova, G. R., Acta mater., 1997, 45, 3687.
22. Mecking, H., Hartig, C. and Kocks, U. F., Acta mater., 1996, 44, 1309

23. Kishida, K., Inui, H. and Yamaguchi, M., Phil Mag. $A$ (in press).

24. Lequeu, P., Gilormini, P., Montheillet, F., Bacroix, B. and Jonas, J. J., Acta metall., 1987, 35, 439.

25. Koeppe, C., Bartels, A., Seeger, J. and Mecking, H., Metall. Trans. A, 1993, 24A, 1795.

26. Umakoshi, Y. and Nakano, T., Acta mater., 1993, 41 , 1155.

27. Lebensohn, R. A., Turner, P. A. and Canova, G. R. Comp. Mater. Sci., 1997, 9, 229.

\section{APPENDIX A}

The viscoplastic potential is a function $F\left(\sigma_{j}{ }^{\prime}\right)$ such as

$$
\dot{\varepsilon}_{j}=\frac{\partial F\left(\sigma_{j}^{\prime}\right)}{\partial \sigma_{j}^{\prime}} .
$$

Therefore, $\dot{\varepsilon}_{j}$ is perpendicular to an equipotential surface, defined by

$$
F\left(\sigma_{j}{ }^{\prime}\right)=F_{0}
$$

where $F_{\mathrm{o}}$ is a reference state.

In the case of a single crystal (see equation (1)) it is easy to see that $F$ is given by

$$
F\left(\sigma_{j}{ }^{\prime}\right)=\dot{\gamma}_{0} \sum_{s} \frac{\tau_{0}^{s}}{n+1}\left(\frac{m_{j}^{s} \sigma_{j}{ }^{\prime}}{\tau_{0}^{s}}\right)^{n+1} .
$$

From equation (A3) follows that $F\left(\sigma_{j}{ }^{\prime}\right)$ is homogeneous of degree $(n+1)$, i.e.

$$
F\left(\lambda \sigma_{j}{ }^{\prime}\right)=\lambda^{n+1} F\left(\sigma_{j}{ }^{\prime}\right)
$$

If the applied stress is a vector $\sigma_{j}{ }^{\left({ }^{(a p p)}\right.}$ lying in a given direction and with an arbitrary modulus, let the corresponding potential be designated as $F_{1}$ :

$$
F\left({\sigma_{j}}^{\left({ }^{(\mathrm{app})}\right)}\right)=F_{1} .
$$

The stress state in the same direction but at the reference potential $F_{\mathrm{o}}$ is given by

$$
{\sigma_{j}}^{\prime(\mathrm{ref})}=\alpha{\sigma_{j}}^{\prime(\mathrm{app})}
$$

where

$$
\alpha=\left(\frac{F_{0}}{F_{1}}\right)^{1 /(n+1)}
$$

